\title{
De la división sexual del trabajo hacia la redefinición de las prácticas de cuidado: una experiencia de Economía Solidaria en Cataluña
}

\author{
From the sexual division of labor to the redefinition of care practices: an \\ experience of Solidarity Economy in Catalonia
}

\author{
Daniela Osorio-Cabrera \\ Universidad Autónoma de Barcelona \\ Departamento de Psicología Social \\ Grupo Fractalidades en Investigación Crítica (FIC)
}

(Rec: mayo 2013 - Acep: junio 2013)

\begin{abstract}
Resumen
El presente artículo surge de la investigación realizada en una cooperativa de la red de Economía Solidaria en Cataluña, en el cual analizamos los cambios y repeticiones en la construcción de significados de los roles de género asociados a la división sexual del trabajo, enfatizando los aspectos relativos a las prácticas de cuidado. Para ello, realizamos una serie de entrevistas a informantes calificados/as del grupo, poniendo énfasis en sus trayectorias laborales. En los resultados se destaca la flexibilización de los roles de género mediante la diversificación de tareas en la cooperativa; ello no limita la reiteración de dinámicas tradicionales de atribuciones de género en lo relativo a tareas reproductivas, aspecto no considerado ni asumido por el colectivo. Sin embargo, la cooperativa se aprecia como un espacio multiplicador de experiencias de cuidado, que promueven la circulación del afecto como un rasgo que se instala y cualifica las relaciones, facilitando la redefinición de las atribuciones de género en el despliegue de tareas.
\end{abstract}

Palabras clave: trabajo, género, economía solidaria, cuidados.

\begin{abstract}
This article emerges from a research conducted in a cooperative of Solidarity Economy network in Catalonia, in which we analyze the changes and repetitions in the construction of meanings of gender roles associated with the sexual division of labor, emphasizing aspects of care practices. We perform a series of interviews with qualified informants of the group, emphasizing their working careers. The results highlight the flexibilization of gender roles through diversification in the cooperative tasks; this does not limit the dynamic reiteration of traditional gender attributions regarding reproductive tasks, something not considered or assumed by the group. However, the cooperative is seen as a space that serves to multiply care experiences that promote the circulation of affect as a feature that is installed and which qualifies relationships, facilitating the redefinition of gender attributions in deployment of tasks.
\end{abstract}

Key words: work, gender, solidarity economy, care 


\section{Introducción}

Con Economía Solidaria (en adelante ES) se ha entendido una forma de nombrar una serie de emprendimientos colectivos que emergen en la década del ochenta en Latinoamérica y del noventa en Europa ${ }^{1}$ (también llamada Economía Social y Solidaria). Se trata de experiencias asociativas dispersas -con distintos grados de formalidad, localización y actividad- que comienzan a gestarse con la intención de promover alternativas socio-económicas a la modalidad capitalista. Adoptan como características centrales: el establecimiento de relaciones horizontales entre sus miembros, el carácter eco-sostenible de sus actividades y la disposición en red, permitiendo la conexión de diversos emprendimientos a nivel territorial y adquiriendo, asimismo, dimensión internacional. Desde la academia se ha teorizado desde hace algunos años sobre su carácter particular y distintivo (por ejemplo, Mugarra, 2004; Laville, 2004; Cruz, 2006).

En las últimas décadas, grupos de economistas feministas han realizado acérrimos cuestionamientos respecto al papel de los mercados como ámbito de la organización social, así como sobre la visión androcéntrica que domina en la economía (Orozco, 2006). Una de las críticas centrales que realizan refiere a la falta de reconocimiento que han tenido históricamente el trabajo doméstico y de cuidados en la esfera económica (Carrasco, 2003; 2011). Dichas tareas han sido naturalizadas en el rol femenino y su invisibilidad ha posicionado a las mujeres en una situación de desventaja. En estas perspectivas se amplía el concepto de trabajo colocando en el centro los modos de vivir en sí mismos, su sostenibilidad y reproducción. Ello implica pensar en la multidimensionalidad de las necesidades, que no se centran solamente en los bienes materiales, sino también los afectos y la cualidad de las relaciones (Carrasco, 2003).

En esta línea, varias investigaciones ${ }^{2}$ (Millán \& Melián, 2008; Orquiza \& García, 2009, Caracciolo \& Foti, 2010, Castañeda, Ascanio, Carosio \& Alva, 2010; Cortes, Lotta, Moreno \& Parada, 2011) tratan de analizar el impacto que las experiencias de ES adquieren en la forma de revalorizar no sólo el papel de las mujeres dentro de la actividad productiva, sino también del rol social de las tareas de reproducción.

\footnotetext{
1 Este trabajo no desconoce la presencia de la Economía Solidaria en otros contextos, pero en relación a los propósitos de esta investigación estos no serán abordados.

2 Cabe destacar que la mayoría de las investigaciones emergen en el contexto latinoamericano.
}

La mayoría de estos trabajos se realizan en experiencias protagonizadas exclusivamente por mujeres, con poco desarrollo en el abordaje de experiencias mixtas. En relación a experiencias protagonizada por mujeres, hay quienes dan un paso más en el análisis, visualizando a la ES como una economía de cuidados, reconociendo en sus prácticas el valor de este tipo de tareas por sobre aquellas que omiten la consideración de la reproducción de la vida humana y la ecología (Burns, 2007; Quiroga, 2009). Consideran también que se promueve en estas prácticas la concepción del trabajo doméstico y de cuidados como responsabilidad colectiva, una distribución equitativa entre hombres y mujeres.

En relación al análisis de la situación de las mujeres en la ES desde mirada(s) feminista(s), encontramos posiciones encontradas. Así, por un lado, se encuentran quienes identifican la ES con planteamientos de este tipo, destacando el acuerdo en ciertos puntos: la satisfacción de las necesidades humanas; el quiebre de las jerarquías económicas opresivas de todos los tipos; el desarrollo del potencial humano; la preservación de las comunidades y el medio ambiente (Matthaei, 2010). Por otro lado, están quienes consideran que la ES puede terminar siendo solo un amortiguador dentro del sistema, corriendo el riesgo de mantener en los márgenes de la economía a un importante número de mujeres (Foufelle \& Palmieri, 2006). Asimismo, coinciden en señalar la necesidad de profundizar en el estudio de estas experiencias, pues visualizan su potencial para la construcción de formas de socialización más igualitarias (Matthaei, 2010; Foufelle \& Palmieri, 2006).

Por todo lo dicho, se ha desarrollado un estudio orientado a identificar en un colectivo de carácter mixto de ES en Cataluña, transformaciones e iteraciones en las percepciones sobre los roles en la división sexual del trabajo. En particular, nos preguntamos acerca de las transformaciones posibles que puedan generar estos espacios en la percepción de los roles de género en la tarea y en el aporte a la conformación de nuevas relaciones sociales, considerando que estos colectivos sostienen en sus principios el establecimiento de relaciones laborales horizontales y de solidaridad. Para realizar el estudio seleccionamos un grupo de la red de ES de Cataluña: la cooperativa de mensajería y limpieza Trèvol. Realizamos seis entrevistas en profundidad con informantes calificados/as, integrantes de la cooperativa, poniendo énfasis en sus trayectorias laborales y valoraciones en relación a nuestro tema de estudio, empleando como técnica de análisis el análisis de contenido. 


\section{Aproximación teórica}

Para realizar este trabajo, consideramos la división sexual del trabajo como enmarcada por relaciones de poder. En este sentido, los últimos trabajos de Foucault (1994/1999) nos proponen pensar mediante dinámicas relacionales los entramados de poder que se despliegan en diversos ámbitos sociales, considerando que se establecen y/o asientan ciertos procesos de dominación, rigidizados o inmovilizados, que determinan la preeminencia de un grupo, de un discurso o de unas prácticas por sobre otras. Junto a lo anterior, las prácticas de sí, que consisten en estrategias que desarrollan y actualizan los sujetos, mediante las cuales "intenta elaborarse, transformarse y acceder a cierto modo de ser" (Foucault, 1994/1999, p. 394).

La división sexual del trabajo ha establecido determinados lugares a hombres y mujeres, prescribiendo prácticas y naturalizado espacios de dominación. En particular, ello ha implicado colocar a las mujeres en situación de subordinación. Esta distinción no conlleva caer en generalizaciones ni esencialismos, en tanto reconocemos que ambas categorías -hombres y mujeresexponen construcciones "que petrifican la realidad del proceso-de-ser-lo-humano-en-el-mundo" (Izquierdo, 2001, p.9). El uso del género como aproximación analítica, nos permite identificar aquellos modelos pre-establecidos que limitan nuestras potencialidades e intereses en las prácticas cotidianas (Amigot, 2005; Izquierdo, 1985).

Ahora bien, suele acontecer que los análisis sobre el género consideran predominantemente los procesos de producción de roles en torno a "la mujer", evaluando estratégicamente el establecimiento y vías de transformación de las posiciones de poder. Con ello se sitúan ciertos binarismos presentes en el análisis social y económico, implicados en funciones como producción/ reproducción, razón/emoción en que participa la dicotomía masculino/femenino, según tales correspondencias (Orozco, 2006). Si bien éstos nos permiten pensar en las generalidades de ciertas situaciones y posiciones de dominación, también nos limitan en la aproximación a otros elementos que circulan en las relaciones entre sujetos: microrrelaciones o formulaciones de rol. Por ello, en este trabajo hemos propuesto analizar los roles de género, pero enfatizando aquello que se construye y distribuye en el espacio intersubjetivo, en tanto representa un "espacio de posibilidad para la transformación de las relaciones de poder" (Amigot \& Pujal, 2009, p. 136).
En este sentido, introducimos también la dimensión afectiva en el análisis de las relaciones sociales, intentando establecer un movimiento de integración epistémico, desde ámbitos en psicología que escinden lo mental de lo corporal, y el intelecto de lo emocional (Sawaia, 2000). Esta tendencia se ha reproducido también en el análisis económico, así como en estudios de acción colectiva, al considerar como residual todo aquello que refiere al orden de lo emocional y afectivo (Flóres Flóres, 2009). Por ello, en este trabajo abordaremos también lo que se ha considerado como redefinición de los cuidados (Precarias a la deriva, 2004). Si bien, en el uso tradicional, el término cuidado refiere a las prácticas de atención a niños y ancianos, en este estudio lo utilizaremos como significante de todas aquellas prácticas que implican el cuidado del otro y del entorno, orientadas al mantenimiento de la vida y la salud, haciendo énfasis en la circulación del afecto en el trabajo como un elemento a considerar.

En los siguientes apartados presentaremos, el diseño de la investigación, especificando las herramientas utilizadas. Posteriormente, los resultados puestos en discusión con nuestras herramientas teóricas e investigaciones que han abordado el tema de estudio. Por último, señalaremos algunas consideraciones finales que intentan resumir las ideas principales del trabajo, así como posibles líneas de indagación a futuro.

\section{Método}

Se realizó un estudio de tipo cualitativo, aproximación que nos permite comprender las singularidades del fenómeno a estudiar, a partir de las vivencias y significados que los sujetos adjudican a sus prácticas. Analizamos las percepciones y valoraciones en torno a las experiencias de trabajo en ES, identificando las iteraciones y transformaciones en relación a los roles de género en la división sexual del trabajo.

\section{Acceso al campo de estudio}

Realizamos un trabajo exploratorio buscando información sobre la presencia de colectivos de ES en Cataluña, tomando contacto con la XES (Xarxa de Economía Sòlidaria de Catalunya o Red de Economía Solidaria de Cataluña). Allí conocimos una cooperativa mixta de trabajo, que integra la red, cuyas actividades principales son la mensajería y limpieza (ambas tareas 
con la particularidad de ser eco-sostenibles). En la selección de esta experiencia influyó el tipo de actividad que desempeñan, considerando la particularidad de que ambos sectores están fuertemente estereotipados en términos de género.

\section{Breve descripción de la organización}

La cooperativa Trèvol es una cooperativa de trabajo que se fundó en el año 1984. En sus inicios funcionaba solamente como mensajería. Surgió a partir de un colectivo de trabajadores que decidieron organizarse, tras una fuerte lucha sindical por mejorar las condiciones laborales y ser despedidos de sus puestos de trabajo; historia sindical que va a marcar el espíritu de sus inicios. Un momento significativo en la historia de la cooperativa, refiere a la decisión de realizar mensajería sostenible, convirtiéndose en la primera empresa de mensajería ecológica de España.

En el año 1994, pasó a fusionarse con una cooperativa de limpieza, la cual también adquiere el carácter ecológico en sus servicios. Esta característica los lleva a organizarse dentro de la REAS (Red de Economía Alternativa y Solidaria). Además conforman la Federación de Cooperativas y es a partir de ésta última, y a iniciativa de un grupo de cooperativistas de la Federación, que Trèvol pasa a constituir con otra serie de organizaciones, la XES. Actualmente la cooperativa está integrada por aproximadamente 50 trabajadores y trabajadoras. Del total, sólo 13 son socios/as de la cooperativa, organizándose en comisiones de trabajo y asambleas. El resto son trabajadores/as contratados/as.

\section{Selección de la muestra}

Los criterios que utilizamos para la selección de la muestra fueron: la pertinencia en relación a la propuesta de estudio; la heterogeneidad -considerando la complejidad, diversidad y número de variables involucradas en el fenómeno-; por último, la accesibilidad y tiempo para el desarrollo del estudio (Reyes \& Hernández, 2008). En relación a la pertinencia, buscamos una experiencia que cumpla con las características que hemos delimitado en relación a las prácticas de ES, un experiencia productiva que nos permitiera analizar la distribución de roles de género en el trabajo; por ello intentamos enrolar en la investigación un colectivo mixto. Para la selección de los entrevistados/as y satisfacer el criterio de heterogeneidad, contemplamos una serie de variables: tratamos de integrar hombres y mujeres de distintas edades que trabajaran en ambos grupos; consideramos estratégica la elección de integrantes del colectivo que se encuentren en distintos momentos de incorporación al mismo, así como la presencia de socio/ as y trabajadores/as contratados/as de la cooperativa. Presentamos en la Tabla 1 con las características de los entrevistados.

\section{Técnicas de recolección de la información}

Como técnica de recolección de información utilizamos la entrevista en profundidad (Taylor \& Bogdan, 2010; Ruíz, 2007), estrategia que nos permitió el acercamiento a los cambios y repeticiones en relación a las formas de significar nuestro tema de investigación.

Tabla 1.

Composición de la muestra.

\begin{tabular}{llcccc}
\hline Entrevistado/a & \multicolumn{1}{c}{ Tarea } & $\begin{array}{c}\text { Tiempo que trabaja } \\
\text { cooperativa }\end{array}$ & $\begin{array}{c}\text { Vínculo } \\
\text { c/ cooperativa }\end{array}$ & Sexo & Edad (años) \\
\hline A & $\begin{array}{l}\text { Mensajería/ } \\
\text { secretario XES. }\end{array}$ & 20 años & Socio & M & 50 \\
B & $\begin{array}{l}\text { Mensajería/ } \\
\text { limpieza }\end{array}$ & 7 años & Socia & F & 42 \\
C & Mensajería & 12 años & Socia & F & 45 \\
D & Limpieza & 2 años & Contratada & F & 32 \\
F & Mensajería & 6 años & Socio & M & 42 \\
G & Limpieza & 3 meses & Contratado & M & 35 \\
\hline
\end{tabular}


Nos focalizamos en aquellos aspectos de la persona que refieren a su trayectoria por distintas experiencias de trabajo, así como en aspectos relativos a las tareas domésticas y de cuidado. Tomamos como referencia el antes y después de su incorporación al colectivo de ES. Elaboramos un guión de entrevista, considerando el desplazamiento progresivo de temas más generales hacía temas específicos en los que pretendíamos profundizar. Tuvimos una entrevista con cada una de las personas seleccionadas, de aproximadamente una hora cada una, siendo la modalidad de registro la grabación de audio. En cada encuentro, y previo a realizar la entrevista, se le explicó a todas las personas los alcances de la investigación, temática, así como la garantía de la confidencialidad de la información recogida. Firmando, posterior a la lectura, un consentimiento informado presentado.

\section{Técnicas de análisis de la información}

Como técnica de análisis, utilizamos el análisis de contenido. Si bien existen diferentes tipos de análisis de este tipo, empleamos como referencia del análisis categorial temático desarrollado por Vázquez (1996), "orientado a temas que provienen de las declaraciones manifiestas y directas" (p. 50). El procedimiento que utilizamos consta de tres etapas: pre-análisis, codificación y categorización. Para el pre-análisis realizamos un ordenamiento de la información, según ejes de análisis pre-establecidos, cuya guía fundamental fueron los objetivos de la investigación. Realizamos una lectura sucesiva de las entrevistas transcriptas, lo cual nos permitió establecer los criterios operativos de análisis. En la codificación transformamos los datos brutos en datos útiles para el análisis, fragmentando el texto y catalogando las unidades. Por último en la $\mathrm{ca}$ tegorización, condensamos la información, agrupando las unidades según criterios de afinidad o analogía; el análisis temático se realiza habitualmente a partir de su significado. Presentamos las categorías y subcategorías en la Tabla 2.

\section{Resultados y discusión}

Dividimos la discusión en tres partes: la primera surge de los resultados obtenidos en la segunda categoría construida, analizando las valoraciones en relación a los roles de género en el trabajo en la cooperativa; la segunda, se centra en los roles de género en el ámbito doméstico; la tercera, trata de un análisis transversal que se fundamenta en los resultados de la primera categoría acerca de las valoraciones de la tarea en la cooperativa. Esta última, habilitó superar algunas dicotomías, en particular en relación al género, reflexionando acerca de los elementos que circulan en las relaciones entre los/as trabajadores/as.

Tabla 2.

Categorías y subcategorías emergentes del análisis.

A.1. Valoraciones sobre el trabajo en la cooperativa

A. Trabajo en la cooperativa

A.2. Tensiones, dificultades en el mantenimiento del colectivo

A.3. Cambios percibidos en la tarea productiva y en la gestión, entre trabajos anteriores y el actual.

B.1. Valoraciones generales acerca de los roles de género en la tarea productiva.

B. Roles de género en la gestión del trabajo en la

B.2. Valoraciones del trabajo de mujeres en mensajería cooperativa

B.3. Valoraciones del trabajo de hombres en limpieza

B.4. Cambios percibidos en relación a experiencias anteriores, acerca de roles de género.

C.1. Valoraciones generales en relación a las tareas domésticas

C.2. Percepciones en relación al reparto de las tareas domésticas

C. Roles de género en las tareas domésticas

C.2.1. Cómo se distribuyen

C.2.2. Factores que influyen en la distribución 


\section{De la fijación de roles de género a su flexibilización en la cooperativa}

Acerca de cómo las personas entrevistadas visualizan la distribución de tareas y la valoración en relación al trabajo realizado por hombres y mujeres dentro del colectivo, la mayoría coincide en plantear el establecimiento de relaciones horizontales entre sus integrantes. No encuentran diferencias en la tarea, según sea realizada por hombres o mujeres, tal como explicita una de las personas entrevistadas.

Eso en lo tangible, y a nivel de trabajo es igual, tampoco en este sentido no estoy de acuerdo en las generalizaciones, es que las mujeres este tipo de cosas las hacen mejor, es que los hombres esta otra lo hacen mejor, yo no creo que vaya por género, la cosa va por individuos. (F)

Sin embargo, encontramos algunos elementos que nos hablan de un "trato diferencial" hacia las mujeres en la cooperativa, tal como plantea una de las integrantes acerca de su participación en el consejo rector (uno de los espacios de gestión de la cooperativa).

Pero... eso sí que hará un par de años que tendría que estar fuera pero continuó por eso, porque soy mujer y para que haya una representación femenina en ese organismo digamos, que es el que decide muchas cosas. (C)

Cuando pasamos a analizar las tareas por separado tanto de mensajería como limpieza, empezamos a encontrar algunos elementos que van en el camino de la flexibilización de los roles de género en la cooperativa, aunque en cierto sentido también persiste una visión sexista en su análisis. Si consideramos las visiones en relación al trabajo en mensajería y en particular al trabajo de mujeres en ese espacio (siendo que la mayoría son hombres), las personas entrevistadas coinciden en señalar que esta situación se debe a la falta de interés de las mujeres en este tipo de tareas, y a un autoreconocimiento de una falta de capacidades para su desempeño, tal como plantea una de las entrevistadas:

Yo creo que hay mujeres que piensan que no van a poder hacerlo o quizás hay mujeres que, no sé, quizás (.) Yo tengo amigas que no les gusta el tema de conducir, porque te estresa mucho, y también estar todo el día en la calle es verdad que es un poco agobiante. Pero... no sé, supongo que debe ser por eso o consideran que no es un trabajo para mujer y ya ni se presentan. (C)

Algunas de las explicaciones que encontramos, refieren a entender que la tarea es visualizada como peligrosa y exigente en términos de fuerza física, rasgos que identifican como masculinos y que afectan el acercamiento de las mujeres.

Sí, sí, porque al final es... una tarea de riesgo, ir por las calles de Barcelona, a veces con mucha prisa. El otro día me estaba comentando [se refiere a su compañera que trabaja en mensajería] que sentía que había algo de parte suya, femenina, que la estaba perdiendo un montón, por tener que coger un montón de su lado masculino para estar en este trabajo. (D)

Encontramos en estas expresiones, cierta forma de comprender lo femenino (prudencia, debilidad) y lo masculino (riesgo, fuerza), imágenes que aparentemente naturalizadas, "condicionantes que nos limitan e imponen una cierta representación de corporalidad, formas de estar que reproducen las normas sociales hegemónicas" (Amigot \& Pujal, 2009, p. 139). Aun así, hay mujeres que se presentan a realizar tareas en mensajería, en particular una de las mujeres entrevistadas, quien considera que las limitaciones mencionadas anteriormente no consisten en limitantes, manifestando ser capaz de hacerlo y que el ambiente de la cooperativa colabora en ese sentido: "Pues yo no sé si será por mi manera de ser, pero yo no me siento diferente por ser::: por lo menos aquí en este trabajo, yo no me siento diferente por ser una mujer" (C). Encontramos en estas manifestaciones algunos elementos que nos hablan de desplazamientos en los roles normativizados, un movimiento práctico desde un lugar de fragilidad o pasividad asociado a lo femenino, valorando las potencialidades singulares en relación a la tarea. Capacidad de acción que permite ejercer nuevas posiciones estratégicas, facilitadas por espacios como la cooperativa, en un ejercicio progresivo de agencia (Amigot, 2007).

$\mathrm{Si}$ pasamos a considerar las valoraciones en relación al trabajo en limpieza, la situación se invierte. En este caso participan en la actividad más mujeres que hombres. En cambio, las consideraciones en relación al por qué, se centran en la discriminación que sufren los hombres en los puestos de trabajo, en particular en el trabajo doméstico, ya que es la sociedad la que no acepta -según dicen- que esta tarea sea realizada por hombres. 
Claro, recuerdo a... [nombra a la encargada de limpieza] que con uno de limpieza, tuvo que explicarles a la empresa, como si tuviera que explicarles, "uy, qué, raro", hombre es que es un sector que tradicionalmente acepta chicas en limpieza. Todas las generaciones de mis padres, de personas que hagan la limpieza doméstica, esperan una chica. $(\mathrm{F})$

Esta valoración es señalada por un trabajador que desarrolla tareas de limpieza, quien plantea diferencias culturales que influyen en estos prejuicios en relación a la distribución de roles y particularmente a la presencia masculina en el trabajo doméstico, sobre todo en el Estado Español. Éste reconoce en la cooperativa la primera oportunidad para acceder a ese tipo tareas, ya que existe una falta de ofertas en el sector.

Pero bueno, quizás por su mentalidad pues puedo trabajar en el servicio doméstico, lo cual, aquí en España, y actualmente es muy difícil, no imposible. Es muy difícil que pueda trabajar la figura de un chico... una figura masculina que haga ese servicio en el hogar, que haga el servicio doméstico para una familia en concreto. $(\mathrm{G})$

Estas valoraciones señalan cómo los sistemas normativos continúan condicionando nuestros modos de actuar con relación a las tareas reproductivas3. En este caso cabe considerar cómo ha sido naturalizada la figura femenina en relación a las tareas de limpie$\mathrm{za}$, y la resistencia que genera la figura masculina en ciertos espacios. Condicionantes que también refieren a contextos socio-culturales, en los cuales estas matrices parecen más difíciles de cambiar. La mayoría de los entrevistados coinciden en señalar que la tarea de limpieza es poco valorada a nivel social, atravesada además por prejuicios y estigmas, no sólo de género. Esta situación se refleja sobre todo en el trato con algunos clientes, como expresa una de las personas entrevistadas: "A mí es que me da igual, porque por ejemplo con un chico decían, pues hostia migrante, hombre, tal, hay un sexismo dentro del cliente, hay un estereotipo" (A); señalamientos que indican la necesidad de considerar al género como nudo de significados (Orquiza \& García, 2009; Caracciolo \& Foti, 2010), reconociendo los distintos otros aspectos asociados, que incluye también las condiciones de clase o étnicas.

\footnotetext{
3 Comprendemos las tareas reproductivas de modo amplio, vinculándolas predominantemente con las labores de sostenimiento de los espacios cotidianos y de cuidado.
}

En relación a los cambios que identifican en relación a trabajos anteriores y desempeño en el actual, sobre la distribución de tareas, la mayoría de los entrevistados plantea no encontrar grandes diferencias. No obstante, a la hora de describir los tipos de tarea asignados a géneros, prima la presencia en espacios de conducción del poder representados por figuras masculinas, mientras que los espacios de subordinación eran asignados en su mayoría a mujeres.

En ese sentido había una diferenciación, pero no por, pero tampoco se notaba nada sexista, sino que era más del rol de que yo soy el que mando, y da la casualidad, bueno no la casualidad que eran todos chicos. Pero no había una gran diferenciación, más que la mayoría de las secretarias, pero salvo una directiva, yo pasé diez años por ahí y sólo fue una directiva. Siempre habían sido hombres. (A)

Finalizando este punto, encontramos coincidiendo con los planteamientos de Biglia (2005) en su análisis sobre los movimientos sociales mixtos, que si bien existe un ambiente antisexista, se reproduce cierta discriminación de género en su dinámica interna, que no siempre es visualizada, incluso por las propias mujeres. Sistemas normativizados difíciles de cambiar, y que atraviesan incluso experiencias de este tipo. Entendemos también que en la cooperativa se abre, a través de la gestión colectiva y horizontal de las tareas, junto a la apertura a ellas según distintos perfiles de acceso, un espacio de trabajo que permite flexibilizar los roles de género preestablecidos. Ello lo expresa una de las integrantes de la cooperativa:

Y... eso, o sea porque no están estrechos de vistas, creo, van creando, brindando oportunidades a la gente, hombres o mujeres de hacer cosas que a veces... Yo creo que también a los hombres les pueden costar un poco el rollo de la limpieza porque no creen que lo hagan bien, o lo que sea. Entonces vienen más mujeres, entonces que no pongan esos límites me parece bien. (D)

\section{En relación a los roles de género en las tareas domésticas y de cuidado}

Acerca de cómo son valorados tanto el trabajo doméstico y como el de cuidados por las personas entrevistadas, nos encontramos con una variedad de 
significaciones que por momentos se contradicen y tensionan. Por un lado, la mayoría considera que es una tarea muy importante, pero poco valorada socialmente. Pero cuando pasamos a denotar cómo son visualizadas en su vida cotidiana, las reconocen como tareas monótonas, poco productivas, que no promueven los niveles de desarrollo personal que les brinda la tarea fuera de la casa: "Pero... a veces sí que se agobia la verdad, a veces está agobiado, porque es muy repetitiva la faena de casa. Yo considero que es muy repetitiva, yo la encuentro muy aburrida." (C). Izquierdo (1998) nos plantea sobre este tema, que la preocupación central en relación a la falta de valoración del trabajo doméstico, no está centrada tanto en la falta de valor en términos de mercado, sino en la falta de aprecio y reconocimiento que recibe el mismo, incluso de la propia persona que lo realiza.

En relación a la distribución del trabajo y del tiempo, las tareas reproductivas aparecen como una limitante para el desarrollo de las actividades en el colectivo, incluso en las productivas, como queda señalado en la historia de una de las entrevistadas, cuya vida laboral estuvo condicionada por las actividades del cuidado.

En el momento que entré aquí me gustó mucho toda la gente que había, eh... el ritmo que se llevaba en todos los aspectos, yo empecé haciendo... me parece que cuatro horas por la mañana y luego ya poco a poco pues ya me fui... mis hijos se fueron haciendo más grandes, y me fueron aumentando horas. (B).

O para invertir el razonamiento, a la hora de considerar el reparto en las tareas domésticas y de cuidado, la mayoría plantea que en la distribución no distinguen si se es hombre o mujer. Cuando pasan a señalar cuáles son los criterios de distribución, la mayoría plantea el tiempo, en relación a las tareas que se desarrollan fuera de la casa. Este argumento coincide con lo que históricamente ha sido una de las excusas para que la mayoría de la tarea recaiga sobre las mujeres, quienes en general trabajan menos horas fuera de casa. Sumado también a los discursos que históricamente han construido a la mujer en relación a la función reproductiva como una de las funciones femeninas normativizadas. No parece ser el caso de la mayoría de las personas entrevistadas; más allá del tiempo, se intentaba buscar un reparto equitativo, que no hiciera recaer las tareas sobre una sola persona. Incluso en algunos casos, la situación se invierte, como lo manifiesta la siguiente persona entrevistada:
Entonces ella tiene muchas horas de trabajo, más horas de trabajo que yo; en ese sentido yo puedo combinarlo más, yo cocino, voy a buscar al crío y lo llevo al cole todos los días y lo voy a buscar tres veces por semana. O sea yo hago mucho más porque tengo más posibilidades de combinarme los horarios que mi compañera. (F)

Identificamos, además, en una de las historias de las personas entrevistadas y en consonancia con una serie de investigaciones que lo plantean (Orquiza \& García, 2009; Caracciolo \& Foti, 2010), la reconfiguración de los roles de género en estas experiencias y su repercusión en términos de autonomía. Nos referimos a una de las entrevistadas que luego de una historia de elecciones laborales marcada en general por las tareas domésticas y de cuidado, pasa a renegociar esta posición en su hogar, en un momento que el trabajo en la cooperativa se lo demandaba.

Entonces... tuvimos una reunión en mi casa, con mis dos hijos y mi compañero y hablamos de que si yo tenía que estar más tiempo y tal, ellos deberían colaborar. Sí sí el que llega antes a casa es el que hace la cena..., o la comida o lo que sea. Es más, yo actualmente, bueno actualmente puedo dedicar más, pero durante cuatro años cuando trabajé en la tienda, llegaba a las nueve y media y cuando llegaba tenía la cena hecha y todo preparado, porque mi compañero llegaba antes y era el que lo tenía que hacer. (B)

La limitación que muchas mujeres experimentan para desarrollar tareas fuera de casa, no es visualizada directamente por casi ninguna de las personas entrevistadas. Salvo una de ellas, que lo plantea en relación a las dificultades que se le presenta a otras mujeres, para participar en instancias de reunión. No parece ser un tema a considerar ni a valorar dentro del colectivo a la hora de pensar estrategias para que todos puedan participar. En esta invisibilidad consideramos que influye el hecho que la mayoría de las personas entrevistadas plantea presentar una distribución equitativa en las tareas de la casa, incluso invirtiéndose los roles tradicionales, con una presencia masculina muy fuerte. Pero también pensamos que puede ser un efecto de la invisibilidad que las tareas domésticas y del cuidado han adquirido históricamente a nivel social y económico (Carrasco 2003; Sassen, 2003), infravalorando la importancia de las mismas en la sustentabilidad de la vida. 
En este colectivo, si bien encontramos una economía que está centrada en la persona y no en el capital, no aparece promovida la división sexual del trabajo reproductivo como un tema de responsabilidad colectiva, tal como ha sido planteado en otras experiencias de ES (Burns, 2007; Quiroga, 2009). Compartimos los planteamientos de Caracciolo \& Foti (2010), quienes proponen la necesidad de que en espacios colectivos mixtos -como la cooperativa-, es necesario trabajar en relación a las corresponsabilidades en las tareas de cuidado doméstico, como una forma de pensar en la distribución del poder al interior de dichos espacios.

\section{La cooperativa como espacio multiplicador de experiencias de cuidado}

En este punto quisiéramos resaltar un elemento que atraviesa nuestro análisis y que, como comentábamos anteriormente, se nos escapa cuando nos centramos exclusivamente en los roles de género. Nos referimos a la noción ampliada de los cuidados, aquellas prácticas en el cuidado de el/la otro/a, que se desarrollan en las actividades cotidianas. En este caso en particular, las que se expresan en referencia a las relaciones laborales dentro de la cooperativa, así como también en el desarrollo de actividades con otras organizaciones que componen la red. En las valoraciones que realizan tanto socios como personas contratadas en relación a la tarea, surgen elementos que nos hablan de la circulación del afecto en el mantenimiento de las relaciones en el trabajo. Situación que se expresa en las palabras de una de las personas entrevistadas:

Yo lo que más destacaría, es el factor humano, el ambiente hay entre los que estamos, que puede haber algún día que te enfades con alguien, yo personalmente no me enfado con nadie. Pero que puede haber algún pique entre socios, pero yo creo que hay un querer, o sea que nos queremos, que si a uno le pasa cualquier cosa, que si uno esta preocupado por algo, los demás también lo están sufriendo. (C)

Afecto que circuló incluso con quienes realizamos la investigación, tanto en la apertura para participar, la generosidad al compartir sus experiencias, y la preocupación por facilitarnos la tarea. Le sumamos como otro elemento a resaltar la generosidad que señalan en relación al intercambio de conocimiento y el aprendizaje entre compañeros/as. Situación que plantean como específica y distinta en relación a la tendencia del ámbito laboral empresarial, donde priman relaciones de competencia. La tarea en la cooperativa apuesta por un intercambio que enriquece a sus integrantes y repercute en el desarrollo de sus potencialidades: "El tema de intercambio de conocimiento en las cooperativa es... que funcionamos con grupos de trabajo, distintos departamentos, es un sentimiento de hacerlo todo en común, hay un intercambio de conocimiento cotidiano." (F)

La confianza, el afecto, la colaboración en la tarea que circula entre quienes comparten un espacio laboral, son elementos no sólo poco valorados en términos económicos, sino también como mencionamos anteriormente, poco destacados a la hora de analizar la acción colectiva desde ámbitos académicos (Flóres Flóres 2009). En un contexto social y laboral en el que se destacan los conceptos de desarrollo individual, autosuficiencia y competencia como estrategias para lograr el éxito, estas experiencias se expresan como una modalidad alternativa que rescata otras formas de relación social, de cuidado. La consideración de el/la otro/a en la tarea, los lazos que se establecen a través de la colaboración y colectivización del conocimiento, van produciendo un cambio de lógica que modula los afectos y que rompe con ciertos marcos normativos previos (Butler, 2010).

Pensar a la cooperativa como un multiplicador de experiencias del cuidado, refiere a que encontramos un espacio de encuentro de experiencias singulares que se articulan, y que pretenden otro tipo de relaciones sociales; revalorizando formas de cuidado que el sistema productivo capitalista deja afuera. Coincidiendo con Hardt \& Negri (2002) acerca de la modalidad alternativa al sistema productivo:

El modo de producción de la multitud es instalado contra la explotación en nombre del trabajo, contra la propiedad en nombre de la cooperación, y contra la corrupción en nombre de la libertad. Auto-valoriza los cuerpos en el trabajo, se reapropia de la inteligencia productiva mediante la cooperación, y transforma la existencia en libertad. (p.353)

Sin duda que este camino no esta exento de tensiones, contradicciones y dificultades que se presentan en las dinámicas de un colectivo, sobre todo a la hora de lograr acuerdos en la gestión de la cooperativa, pero son asumidas también como parte de un proceso: 
A veces puede haber discrepancias, por más que seamos una cooperativa, por muchos que seamos, que tengamos visiones similares, por otro lado puede haber distintos puntos de vista, puede haber una discrepancia, pero forma parte del juego también. Y pues bueno no es lo ideal, lo ideal sería que siempre nos llegáramos entender. Pero sí que se puede producir discrepancias internas, y bueno para eso está la democracia. (F)

La cooperativa se presenta como una propuesta que en el marco de lo micro, intenta articular lo político, económico y social como elementos característicos, y el soporte afectivo como un elemento más a valorar en la forma de actuar en la economía; sin duda uno de los grandes ausentes en este ámbito. Esta cadena de afecto se extiende incluso con otras organizaciones con las que trabajan dentro de la red de ES, compartiendo principios y valores. Situación que se reafirma en las palabras de algunas de las personas entrevistadas al finalizar la entrevista, quienes agradecen la oportunidad de poder trabajar en experiencias de este tipo, y la intención de que ésta sea conocida para que más gente vea que se puede vivir de otra manera. Plantear la ampliación del concepto de cuidado, incluyendo la afectividad en emprendimientos como la cooperativa, implica pensar en la construcción de otro tipo de relaciones, que llevan a descentrar la afectividad de la figura femenina y valorizarlo como parte de las relaciones entre las personas. Coincidiendo con los planteamientos de Sawaia (2001), entendemos la incorporación del afecto en el estudio de la acción social transformadora, como uno de los desafíos a abordar por las ciencias sociales.

\section{Consideraciones Finales}

A través de esta investigación, nos propusimos explorar las experiencias de personas que participan en la ES, analizando en qué medida este tipo de prácticas, ayudan a romper las visiones acerca de roles de género preestablecidos en la división sexual del trabajo; así como su aporte al desarrollo de nuevas relaciones sociales. En este sentido, la mayoría de las personas entrevistadas plantea manejarse en igualdad de condiciones, valorando a la persona en la tarea y no en función de su sexo. Sin embargo, encontramos que se mantienen algunos elementos de análisis sexista y una dificultad en particular para visualizar el reparto de las tareas domésticas y de cuidados como una temática a reflexionar en el colectivo. Fundamentalmente en lo que refiere a un reparto que permita generar igualdad de oportunidades para participar dentro de la organización. Entendemos en este punto, una línea abierta a seguir pensando en estas experiencias.

Visualizamos también el riesgo que implica pensar en términos exclusivamente de género, o en relación a la división sexual del trabajo, porque muchas veces ello implica la reproducción de lógicas binarias en el análisis. Aún así llevamos a cabo esta labor, para utilizarlo en términos operativos y para develar las modalidades relacionales respecto a las atribuciones de rol. En el último apartado de los resultados y las discusiones, incorporamos algunas dimensiones nuevas en relación al cuidado, que nos permitieron en cierto sentido superar estas dicotomías. Este último paso, nos permitió visualizar algunos otros elementos de análisis. Encontramos en estas experiencias un espacio que actúa como multiplicador de experiencias de cuidado: basadas en el cuidado de el/la otro/a y la circulación del afecto en la tarea. Un camino que no está exento de dificultades y contradicciones, pero que lleva a estos sujetos a elegir cotidianamente su labor y a la intención de difundirla como una modalidad alternativa no sólo de producción de bienes o servicios, sino como un modo de vida. $\mathrm{Al}$ hacer este señalamiento, no minimizamos algunas diferenciaciones tradicionales respecto al género que hemos encontrado. Sin embargo, nos parece interesante resaltar la posibilidad y potencialidad que este tipo de experiencias brinda en el sentido de constituir relaciones que buscan la horizontalidad y la circulación del afecto como elemento central en las mismas.

Entendemos que un elemento a seguir abordando en el estudio de estos colectivos, refiere a incorporar la lectura del afecto como potencial transformador. Ello, sobretodo, si estas experiencias pretenden ser alternativas al sistema económico que regula, media y promueve ciertos tipos de relaciones que minimizan el capital de lo afectivo en el establecimiento de vínculos en el ámbito productivo -nos referimos al capitalismo-.

\section{Referencias}

Amigot, P. (2005). Relaciones de poder, espacio subjetivo y prácticas de libertad: análisis genealógico de un proceso de transformación de género. Tesis doctoral. Depto. Psicología Social. UAB.

Amigot, P. (2007). Una tensa oscuridad. Interrogando el abordaje psicosocial de la subjetividad. Psicología \& Sociedade, 19(3), 20-25.

Amigot, P., Margot P. (2009) Una lectura del género como dispositivo de poder. En revista: Sociológica, 24(70) 115-152 . Recuperado de http://www.revistasociologica.com.mx/pdf/7005.pdf 
Amoroso, M; Pareras, A; Carrasco, C; Medrano, H \& Sáenz, N. (2003). Malabaristas de la vida. Mujeres, tiempos y trabajos. Barcelona. Icaria.

Biglia, B. (2005) . Narrativas de mujeres sobre las relaciones de género en los Movimientos Sociales. Tesis Doctoral. Departamento de Psicología Básica. Universidad de Barcelona.

Butler, Judith.(2010). Marcos de Guerra. Las vidas lloradas. Madrid: Paidos.

Bourns, A. (2007). Politizando la pobreza, hacia una economía social del cuidado. Salvador: Progressio.

Caracciolo, M. \& Forti MP. (2010) Las mujeres en la economía social y solidaria: experiencias rurales y urbanas en Argentina. UNIFEM. Recuperado de: http://www.asociacionlolamora.org.ar/ doc/Las-mujeres-en-la-economia-social-y-solidaria-experienciasrurales-y-urbanas-en-argentina.pdf

Carrasco, C. (2011). La economía del cuidado: planteamiento actual y desafíos pendientes. Revista de Economía Crítica, 11, 205-225. Recuperado de: http://www.paralelo36andalucia.com/wpcontent/2011/07/REC11_9_intervenciones_CristinaCarrasco.pdf.

Castañeda, N., Ascanio, C., Carosio, A. , Alva.ME. (2010) . El trabajo socio productivo: En el marco de la economía social y la igualdad y equidad de género. Revista Venezolana de Estudios de la Mujer. 15(35), 59-71. Recuperado de: http://www.scielo.org.ve/ scielo.php?pid=S1316-37012010000200004\&script=sci_arttext

Cruz, A. (2006). A construção do conceito de Economia Solidária no Cone Sul. Revista Estúdios Cooperativos, 12(1), 7-27.

Cortes, C., Lotta. L., Moreno, H. \& Parada, A. (2011) Aproximación a las prácticas organizacionales de la asociación de mujeres recicladoras-Asofilia. Colombia. Universidad Piloto de Colombia.

Flóres Flóres, M. J. (2009). Tácticas de des-sujeción: disensos, subjetividad y deseo en los movimientos sociales: relaciones de género en la red 'Proceso de Comunidades Negras" del Pacífico colombiano. Tesis Doctoral. Departamento de Psicología Social UAB.

Foucault, M. (1994-1999). Estética, ética y Hermenéutica. Barcelona: Paidós.

Foufelle, D. \& Palmieri, J. (2006). Feminismo y economía social y solidaria. Recuperado de: http://www.ciudaddemujeres.com/ articulos/Feminismo-y-economia-social-y

Hardt, M. \& Negri, A. (2002). Imperio. Barcelona: Paidós.

Izquierdo, M.J. (1985) las, los, les (lis, lus) el sistema sexo/género y la mujer como sujeto de transformación social. Barcelona: Ediciones de les dones.

Izquierdo, M. J. (1998). El malestar en la desigualdad. Madrid: Cátedra. S.A.

Izquierdo, M. J.(2001) Sin vuelta de hoja. Sexismo: poder, placer y trabajo. Barcelona: Bellatera.
Laville, J.L. (2004). El marco conceptual de la Economía Solidaria. En J.L., Laville (Ed.), Economía social y solidaria. Una visión europea. Buenos Aires: Altamira,

Mattahei, J. (2010). Más allá del hombre Económico: Crisis Económica, Economía Feminista, y la Economía Solidaria. Revista Venezolana de Economía Social 10(19), 65-80.

Millán, M.G. \& Melián, A. (2008) El mercado de trabajo femenino en las empresas de economía social de Andalucía (España). Un análisis econométrico del perfil de la mujer trabajadora. Revista Pecvnia, 7, 227-257. Recuperado de: http://www3.unileon.es/ pecvnia/pecvnia07/07_227_256.pdf

Mugarra, A. (Ed) (2004) La Economía Solidaria y su inserción en la formación universitaria. Bilbao: Editorial de la Universidad de Deusto.

Orquiza. C., García, M. A.(2009). Aspectos transformadores y obstáculos a superar: práctica social y educativa de mujeres en la Economía Solidaria. Revista de Estudios Experiencias en Educación, 8(15), 11-29. Recuperado de: http://redalyc.uaemex.mx/ redalyc/pdf/2431/243116377001.pdf

Quiroga, N. (2009) Economías feminista, social y solidaria. Respuestas heterodoxas a la crisis de reproducción en América Latina. Iconos. Revista de Ciencias Sociales, 33, 77-89. Recuperado de: http://redalyc.uaemex.mx/src/inicio/ArtPdfRed. jsp?iCve $=50903307$

Precarias a la Deriva, (2004). A la deriva por los circuitos de la precariedad feminina. Madrid: Traficantes de Sueños.

Reyes. P. \& Hernández, A. (2008). El Estudio de Caso en el contexto de la Crisis de la Modernidad. Cinta Moebio, 32, 70-89 Recuperado de: http://www.scielo.cl/pdf/cmoebio/n32/art01.pdf

Ruíz Olabuénaga, JI. (2007) Metodología de la investigación cualitativa. España, Eitorial Universidad de Deusto.

Santos, B. (2005) El milenio huérfano. Ensayos para una nueva cultura política. Madrid: Trotta.

Sassen, S. (2003). Contrageografías de la globalización. Género y ciudadanía en los circuitos transfronterizos. Madrid. Traficantes de Sueños.

Sawaia, B. (2000). A emoção como locus de produção do conhecimento. Uma reflexão inspirada em Vygotsky e no seu diálogo com Espinosa. III Conferencia de pesquisa Socio Cultural. 19-20 de julio de 2000. Campinas, Brasil. Recuperado de: http://es.scribd. com/doc/38652486/A-emocao-como-locus-de-producao-doconhecimento-Uma-reflexao-inspirada-em-Vygotsky-e-no-seudialogo-com-Espinosa.

Taylor, S. \& Bodgan, R. (1992). Introducción a los Métodos Cualitativos. Paidós: Barcelona.

Vázquez, F. (1996). El análisis de contenido temático. Objetivos y medios en la investigación psicosocial. (Documento de trabajo) (pp. 47-70). Universitat Autònoma de Barcelona. 